\title{
Ciprofloxacin Induced Chondrotoxicity and Tendinopathy
}

\author{
Elias Adikwu and Nelson Brambaifa \\ Department of Pharmacology, Faculty of Basic Medical Sciences, \\ College of Health Sciences, University of Port Harcourt, Rivers State Choba, Nigeria
}

\begin{abstract}
ABSRACT
Ciprofloxacin is one of the fluoroquinolones with a wide clinical acceptability. Rescently there are increasing reports on Ciprofloxacin induce Chondrotoxicity and Tendinopathy in Animal experiment and clinical experience which is of great clinical concern. A comprehensive survey and review of literaure on reported ciprofloxacin induced Chondrotoxicity and Tendinopathy in Humans and Animals was performed. It was observd that ciprofloxacin is a potential inducer of Chrondrotoxicity and Tendinopathy which could be potentiated by coadministration with corticosteroids. This conditions were reported to be characterised by cartilage lesion, matrix swelling, inhibition of chondrocytes proliferation, secretion of soluble proteoglycan, modification of the metabolism and integrity of extracellular proteins, decrease in epiphyseal growth plate, humerus and femur.The mechanism behind this phenomenon is said to be multifactoral. Ciprofloxacin induced Chrondrotoxicity and Tendinopathy in growing animals is attributed to oxidative stress (lipid peroxidation, Deoxyribonucleic Acid (DNA) oxidative stress). Ciprofloxacin induced cartilage damage may also be attributed to formation of Ciprofloxacin chelates and complexes which possesses the potential to induce a deficiency of functionally available divalent ions resulting in cytoskeletal changes. Animal studies showed that oxidative damage or metabolism of tissues was also found suggesting the involvement of a reactive oxygen species. Administration of magnesium, zinc chloride and vitamin $\mathrm{E}$ ( $\alpha$ tocopherol) were found to prevent or reverse ciprofloxacin induced Chrondrotoxicity and Tendinopathy. Through excess formation of collagen, increase osteoblastic activity, increase bone growth, inhibition of free oxidation radicals' formation thereby preventing DNA oxidation and oxidative stress. Zinc also directly stimulates DNA synthesis either by enzyme stimulation or altering, the binding of $\mathrm{fl}$ and $\mathrm{f} 3$ histones to DNA so as to affect RNA synthesis. Patient medical history should be considered before Ciprofloxacin recommendation. Coadministration with corticosteroid should be done with caution. Further evaluation of antioxidants effect in Ciprofloxacin induce Chonrotoxicity, Tendinopathy in humans could be of clinical importance as observed in Animal studies.
\end{abstract}

Keywords: Ciprofloxacin, Chrondrotoxicity, Tendinopathy, Tendon Rupture

\section{INTRODUCTION}

The first Quinolone, nalidixic acid was first isolated as a byproduct of the synthesis of chloroquine in early 1960s (Hall et al., 2011) Later in the 1980s fluorinated derivatives e.g., ciprofloxacin were synthesized (Stahlmann, 2002). Ciprofloxacin is a second generation fluoroquinolone with a broad spectrum of antibacterial activity. It has a good bioavailability after oral administration, good to excellent tissue penetration and relative safe (Ball and Tillotson, 1995; Papich, 1998). It is very active against wide variety of pathogenic bacteria including some gram-positive and most grain-negative organism (Hooper and Wolfson, 1985). It is used in a variety of human clinical infections (Sub and Lorber, 1995).

Ciprofloxacin exerts its action by blocking bacterial DNA synthesis through inhibition of bacterial topoisomerase ii (DNA gyrase) and topoisomerase iv. Inhibition of DNA gyrase prevents the relaxation of positively super coiled DNA that is required for normal transcription and replication (Lica and Zhao, 1997; Alovero et al., 2000; Hooper, 2000). Inhibition of topoisomerase iv interferes with separation of replicated chromosomal DNA into respective daughter cells during cell division (Mitscher and Mao, 2003).

Ciprofloxacin is generally well tolerated and remains one of the safest of all antibiotics with 
remarkably few reactions (Hooper and Wolfson, 1985; Ball, 1986). These reactions include gastrointestinal tract, central nervous system and hematological system (Petri, 2001; Lode et al., 1998).

Despite its safe profile there are reported cases of Ciprofloxacin induced chrondrotoxicity, Tendinopathy and tendon rupture in animals and humans (Khaliq and Zhanel 2003; Channa et al., 2008). It was reported that Ciprofloxacin decreased thickness of the articular cartilage of the femoral condyle, inhibit proliferation of cultivated chondrocytes and secretion of soluble proteoglycans in a concentration and time dependant manner (Li et al., 2004). Ciprofloxacin induced damage of the articular and epiphysical growth plate cartilage of knee joint, tendinopathy and tendon rupture (Halawa, 2010; Kim, 2010).

The pathogenesis of Ciprofloxacin induced chrondrotoxicity, tendinopathy and tendon rupture is a multifactoral event (Halawa, 2010). Previous studies attributed cartilage damage in growing animals to oxidative stress, lipid peroxidation and DNA oxidative damage of the chondrocytes and collagen (Simonin et al., 1999; Li et al., 2004).

Other authors' referred to Ciprofloxacin induced tendinopathy to their inhibitory effects on DNA, collagen and proteoglycan synthesis (Maslanka et al., 2004). As of July 2008, the United State Food and Drug Administration mandated that Ciprofloxacin product should have a black-box warning indicating an increased risk in adverse events including tendon rupture (Kim, 2010). This study reviews reported Ciprofloxacin induced Chrondrotoxicity, Tendinopathy and tendon rupture in humans and animals due to rising trend.

\subsection{Animal Studies}

Experiments with animals have reported cases of Ciprofloxacin induced chrondrotoxicity, tendinopathy and tendon rupture (Li et al.,1990; Stahlmann, 2003; Tsai et al., 2008). It was observed that Ciprofloxacin at a dose level of $20 \mathrm{mg} \mathrm{kg}^{-1}$ day administered to rats for 15 days induced articular damage, cavity Formation wide clefts and decrease in both articular and epiphyseal growth plate (Halawa, 2010). This report is in resonance with the report of Channa et al. (2008) who administered $20 \mathrm{mg} \mathrm{kg}^{-1}$ of Ciprofloxacin to wistar albino pulps. He observed decrease in the width of epiphyseal growth plate cartilage, humerus and femur as compared to the control.

In another study where Ciprofloxacin was administered to newly born rat-litters intraperitonealy. Ciprofloxacin induced growth plate retardation by inhibiting mitosis in the proliferative zone. Ciprofloxacin also affected the mean length of humora and femora leading to reduction in Limb length of rat pulps
(Channa et al., 2008). The above reports could be consistent with the study of Stahlmann et al. (2000) who reported that $200 \mathrm{mg} \mathrm{kg}^{-1}$ of Ciprofloxacin administered to immature Beagles induced cleft formation and erosion of joint cartilage. Male wistar rats exposed to $50 \mathrm{mg} \mathrm{kg}^{-1}$ of Ciprofloxacin for 21 days inhibit fracture healing with decreased cartilage cellullarity and fibrous proliferation and matrix degeneration (Huddleston et al., 2000).

Furthermore, 30 and $90 \mathrm{mg} \mathrm{kg}^{-1}$ oral doses of Ciprofloxacin was found to induce a characteristic arthropathy (blister and erosions) in Juvenile beagle with persisted lesions after five (5) months treatment free period (Keutz et al., 2004). Ciprofloxacin in a 2 weeks study induced cartilage blisters when 100 and $200 \mathrm{mg} \mathrm{kg}^{-1}$ were administered to Juvenile dogs. In another study, cartilage alterations in Knee joints were seen after $30-100 \mathrm{mg} \mathrm{kg}^{-1}$ of Ciprofloxacin was administered for 3 weeks (Schuter, 1987). Several cartilage lesions marked by matrix swelling and loss of chondrocytes were observed when 400, 800 and $1200 \mathrm{mg} \mathrm{kg}^{-1}$ of Ciprofloxacin was administered to 4 week-old rats for 7 days consecutively. The thickness of the femoral condyle was significantly decreased compare to the control. Proliferation of chondrocytes and secretion of soluble proteoglycans were also inhibited (Li et al., 2004; Pfister et al., 2007).

Other authors found that single high oral doses and multiple low doses of Ciprofloxacin were chondrotoxic in Juvenile rats. Ciprofloxacin induced scars and erosions of the joint surface as well as chondrocytes clusters with non Cellular areas of the articular matrix (Foster et al., 1997; Stahlmann, 2003). The study of Li et al. (2004) is in support of the above report. He reported that Ciprofloxacin inhibited chondrocytes proliferation in a dose dependant and time dependant manner. $10-80 \mathrm{mg} \mathrm{L}^{-1}$ of Ciprofloxacin decreased secretion of soluble proteoglycans after incubation with chondrocytes for 5 days.

Subcutaneous injection of $600 \mathrm{mg} \mathrm{kg}$ of Ciprofloxacin administered to 34-day old wistar rats induced cartilage lesion in 11 of 12 (92\%) rats (Stahlmann et al., 1999; Pfister et al., 2007). Ultra structural changes were observed in Achilles tenocytes of Ciprofloxacin treated Spraque Dawley rats (Stahlmann, 2002; Bae et al., 2006). These reports are at variance with the study of Kashida and Kato (1997), who observed that $200 \mathrm{mg}$ and $900 \mathrm{~m} \mathrm{~kg}^{-1}$ of Ciprofloxacin administered to rats did not induce Achilles tendon toxicity.

Some researchers observed that Ciprofloxacin induced cartilage damage in experimental animals when administered during certain developmental stages via changes of cytoskeleton morphology (Water et al., 1998; 
Egerbacher et al., 2000). Ciprofloxacin at a dose level of $50 \mathrm{mg} \mathrm{kg}^{-1}$ administered to rats for 3 weeks revealed significant deterioration of Biochemical parameters, hyaline degeneration and fibre disarrangement in the tendon of rats (Olcay et al., 2011).

Findings showed that Ciprofloxacin exerts a negative impact on migration and proliferation as well as the collagen metabolism of tenocytes (Mehra et al., 2004; Tsai et al., 2011). Pauzaud et al. (2004) reported that Ciprofloxacin showed moderate Cytotoxicity after 28 hours and more severe significant toxicity after 72 hours on tendon cells. This agrees with the study which observed that Ciprofloxacin inhibits tendon cell proliferation and cause cell cycle arrest in rats (Tsai et al., 2008). It was reported that cell proliferation in horse and dog chondrocytes decreased in Ciprofloxacin cultivated groups (Egerbacher et al., 2001).

Incubation of Achilles tendon, Achilles paratenon and shoulder capsule fibroblast with Ciprofloxacin hydrochloride (5-50 $\mathrm{pg} \mathrm{mL}^{-1}$ ) significantly decreased cell proliferation, collagen synthesis and proteoglycan synthesis in fibroblast cell line (Williams et al., 2000). Degenerative changes in tenocytes due to swelling and dilatation of cell organelles, densified nuclei and clumped chromatin cell detachment from extracellular matrix, decrease in fibril diameter and increase distance between collagenous fibrils were induced by Ciprofloxacin in immature wistar rats (Shakibaei et al., 2000).

\subsection{Human Studies}

There is increasing trend of Ciprofloxacin induced tendinopathy and tendon rapture in human (McEwan and Davey, 1988; Movin et al., 1997; Short et al., 2006). There is a reported case of Ciprofloxacin associated Achilles tendon diseases. Microscopic evaluation revealed irregular collagen fibre arrangement, hypercellularity and increase interfibrillar glycosaminoglycan (West and Gow, 1998; Pantalone et al., 2011). Ciprofloxacin induced Achilles tendon rupture characterized by degenerative changes in left Achilles tendon, cystic changes with focal necrosis (Hugo-Persson, 1996; Jagose et al., 1996; Petersen and Lapress, 1998).

Khazad and Schwenk (2005) reported a case of non traumatic rupture of Achilles tendon in a patient who received oral Ciprofloxacin $500 \mathrm{mg}$ twice orally for urinary tract infection. This agrees with the report of other authors (Lee and Collins, 1992; Poon and Sundaram, 1997; Caspirian et al., 2000).

In a case study, 32 patents $(76 \%)$ had tendinitis and 10 patents $(24 \%)$ had tendon rupture. 13 (31\%) were attributed to Ciprofloxacin (Linden et al., 2001). This is at variance with a study where no cases of Achilles tendon rupture were found in 2, 122 Ciprofloxacin treated patients (Shinohara et al., 1997). In France Pierfitte et al. (2000) reviewed 421 cases of Fluoroquinolones associated tendinopathy. They reported that only $5 \%$ of Fluoroquinolones associated tendinopathy was attributed to Ciprofloxacin.

In a critical literature review of fluoroquinolones associated tendinopathy in humans. It was observed that Ciprofloxacin is one of the agents associated with tendinopathy (Khaliq and Zhanel, 2003; Mirovsky et al., 1995; Brava et al., 1996; Carrasco et al., 1997). In a case study, 98 cases of fluoroquinolones induced tendon injury were reported. It was observed that the second most commonly implicated fluoroquinolone associated with tendon injury was Ciprofloxacin $(25.5 \%$ of all cases) with total daily doses ranging from $500-200 \mathrm{mg}$ for a mean duration of $24 \pm 29.3$ days (Khaliq and Zhanel, 2003).

In an observed cohort study of fluoroquinolones and other antibiotics induced tendon disorder. It was reported that Ciprofloxacin is also associated with tendon disorder but with effects lesser than that of Ofloxacin (Wilton et al., 1996). In 1795 case report forms for children receiving Ciprofloxacin which were collected up to the end of 1994 by Bayer cooperation Germany (Chysky et al., 1991; Hampel et al., 1997) shows that Ciprofloxacin is safe for children, adolescents and do not have negative effects on the linear growth children. This is at variance with what was observed in animal studies (Kato, 2008).

The confirmation of the safety of Ciprofloxacin especially in relation to the skeletal system in large scale clinical studies has led to it being approved for use in pediatric patients with cystic fibrosis (Kato, 2008). This report could be at variance with the report of Adefurin et al. (2011). Who stated that a total of 16184 pediatric patients who were exposed to Ciprofloxacin and 1065 reported cases of musculoskeletal adverse effects were observed.

Furthermore, monolayers of human tenocytes were incubated with Ciprofloxacin. Ciprofloxacin significantly decreased type 1 collagen, beta (1)-integrin receptors, cytoskeletal and signaling proteins. It increased matrix metalloproteinases as well as the apoptosis marker activated caspase 3 effects are intensified at higher concentrations and longer incubation periods (Corps et al., 2005; Sendzik et al., 2005; 2010).

\subsection{Pathogenesis}

The pathogenesis of fluoroquinolones (Ciprofloxacin) induced chrondrotoxicity, tendinopathy and tendon rupture has not been fully established (Khaliq and Zhanel 
2003). Although a number of suggestions have been made, previous studies attributed articular cartilage damage in growing animals to oxidative stress lipid peroxidation and DNA oxidative damage of chondrocytes and collagen. Those factors collectively resulted in modification of the metabolism and integrity of extracellular proteins (Simonin et al., 1999; Maslanka et al., 2004; Li et al., 2010).

Ciprofloxacin induced chrondrotoxicity was also explain on the basis that Ciprofloxacin chelates magnesium ions or divalent ions resulting in change function of chondrocytes surface integrin receptors (Stahlmann et al., 1995; Shakibaei et al., 2000; Lozo et al., 2004). This report is supported by the ability of magnesium to reverse or inhibit Ciprofloxacin induced chrondrotoxicity (Stahlmann et al., 1999; Stahlmann, 2002). This shows that patients with latent magnesium deficiency could be at an increase risk of Ciprofloxacin induce tendon disorder. Pfister et al. (2007), also supported this view when he demonstrated that Ciprofloxacin induced cartilage lesions was reversed in animals treated with vitamin $\mathrm{E}$ ( $\alpha$ tocopherol) and magnesium or both. This also agrees with other studies (Stahlmann et al., 1999).

It was also reported that Zinc chloride minimized epiphyseal cartilage damaged induced by Ciprofloxacin in wistar albino rats. This agrees with other observations (Channa et al., 2008). Hickory et al. (1979) reported that zinc helps in excess formation of collagen, increase osteoblastic activity and increase rate of longitudinal growth and bone remodeling in experimental rats. Zinc also directly stimulates DNA synthesis either by enzyme stimulation or altering the binding of $f_{1}$ and $f_{3}$ histones to DNA so as to affect RNA synthesis (Prasad, 1991).

Animal studies showed that oxidative damage or metabolism of tissues was also found suggesting the involvement of a reactive oxygen species (ThuongGuyot et al., 1994; Hayem et al., 1996; Khaliq and Zhanel, 2003).

Corticosteroid may precipitate Ciprofloxacin induce rupture of Achilles tendon. The exact mechanism by which corticosteroids cause tendon damage is not clear. It is said that steroids have the ability to alter the collagen structure of tendons by contributing to dysplasia of collagen fibrils, thus reducing the tensile strength of the tendon (Kelly et al., 2004). Corticosteroids can also interfere with collagen fibre cross-linking which can lead to disruption in the normal healing process of the tendon (Orava et al., 1996; Kotnis et al., 1999; Kelly et al., 2004).

\section{REFERENCES}

Adefurin, A., H. Sammon, E. Aigrain and I. Choonara, 2011. Ciprofloxacin safety in paediatrics: A systematic review. Arch. Dis. Child., 96: 874-880. DOI: 10.1136/adc.2010.208843

Alovero, F.L., X.S. Pan, J.E. Morris, R.H. Manzo and L.M. Fisher, 2000. Engineering the specificity of antibacterial fluoroquinolones: Benzenesulfonamide modifications at C-7 of ciprofloxacin change its primary target in Streptococcus pneumoniae from topoisomerase IV to gyrase. Antimicrob. Agent Chemother., 44: 320-325. PMID: 10639357

Bae, C.S., D.M. Oh, J.G. Bae, J.C. Kim and S.H. Kim et al., 2006. Ultrastructural changes of the gemifloxacin on Achilles tendon in immature rats: Comparison with those of ciproxacin and ofloxacin. Basic. Clin. Pharmacol. Toxicol., 98: 406-410. PMID: 16623866

Ball, P. and G. Tillotson, 1995. Tolerability of fluoroquinolone antibiotics. Past, present and future. Drug Saf., 13: 345-358. PMID: 8652079

Ball, P., 1986. Ciprofloxacin: An overview of adverse experiences. J. Antimicrob. Chemother., 18: 187193. PMID: 3542945

Brava, S., M. Hume and J. Leppilahki, 1996. Bilateral Achilles tendon rupture: A report on two cases. Scand J. Med. Sci. Sports, 6: 309-312. DOI: 10.1111/j.1600-0838.1996.tb00476.x

Carrasco, J.M., B. Garcia, C. Andujar, F. Garrote and P.D. Juana et al., 1997. Tendinitis associated with ciprofloxacin. Ann. Pharmacother, 31: 120-120.

Caspirian, J.M., M. Luchi, R.E. Moffat and D. Hinthorn, 2000. Quinolones and tendon ruptures. Med. J., 93: 488-491. PMID: 10832946

Channa, H.M., M. Ashfag, R. Bangash, A. Abbaj and M.A. Qureshi, 2008. Preventive role of zinc chloride against toxicity of ciprofloxacin on the growing cartilage of Wistar albino rat litter. J. Ayub. Med. Coll. Abbottabad, 20: 77-81. PMID: 19999211

Chysky, V., K. Kapila, R. Hullmann, G. Arcieri and P. Schact et al., 1991. Safety of ciprofloxacin in children: Worldwide clinical experience based on compassionate use. Emphasis on joint evaluation. Infection, 19: 289-296. PMID: 1917049

Corps, A.N., R.L. Harrall, V.A. Curry, B.L. Hazleman and G.P. Riley, 2005. Contrasting effects of fluoroquinolone antibiotics on the expression of the collagenases, Matrix Metalloproteinases (MMP)-1 and -13 , in human tendon-derived cells. Rheumatology, 44: 1574-1517. DOI: 10.1093/rheumatology/kei087 
Egerbacher, M., B. Wolfesberger and C. Gabler, 2001. In vitro evidence for effects of magnesium supplementation on quinolone-treated horse and dog chondrocytes. Vet. Pathol., 38: 143-148. PMID: 11280370

Egerbacher, M., G. Seiberl, B. Wolfebergen and I. Walter, 2000. Ciprofloxacin causes cytoskeletal changes and detachment of human and rat chondrocytes in vitro. Arch. Toxicol., 73: 557-563. PMID: 10663387

Foster, C., R. Schwabe, E. Lozo, U. Zippel and J. Vormann et al., 1997. Quinolone-induced arthropathy: Exposure of magnesium-deficient aged rats or immature rats, mineral concentrations in target tissues and pharmacokinetics. Arch. Toxicol., 72: 26-32. PMID: 9458187

Halawa, A.M., 2010. Effect of ciprofloxacin on the articular cartilage and epiphyseal growthplate cartilage in the growing albino rats and the possible protective roleof vitamin E ( $\alpha$-Tocopherol): A histological and morphometric study. Egypt. J. Histol., 33: 569-582.

Hall, M.M., J.T. Finnoff and J. Smith, 2011. Musculoskeletal complications of fluoroquinolones: Guidelines and precautions for usage in the athletic population. PM. R., 3: 132-142. PMID: 21333952

Hampel, B., R. Hullmann and R. Schmidt, 1997. Ciprofloxacin in Pediatrics. Ciprofloxacin in pediatrics: Worldwide clinical experience based on compassionate use--safety report. Pediatr. Infect. Dis., 16: 127-129. PMID: 9002122

Hayem, G., O. Domarle, M. Lay, M. Thuong-Guyot and J.J. Pocidalo et al., 1996. Lack of correlation between hydrogen peroxide production and nitric oxide production by cultured rabbit articular chondrocytes treated with fluoroquinolone antimicrobial agents. Toxicol. Vitro, 10: 551-555. PMID: 20650236

Hickory, W., R. Nanda and F.A. Gatalanolo, 1979. Fetal skeletal malformations associated with moderate zinc deficiency during pregnancy. J. Nutr., 109: 883891. PMID: 438902

Hooper, D.C. and J.S. Wolfson, 1985. The fluoroquinolones: Pharmacology, clinical uses and toxicities in humans. Antimicrob. Agents Chemother., 28: 716-721. PMID: 2936302

Hooper, D.C., 2000. Quinolones. In: Marvell Douglas and Bennetts Principles and Practice of Infections Disease 5th, Mandell, G.L., J.E. Benntt and R. Dolin (Eds.)., Churchhill Livingstone. New York, pp: 403-423.

Huddleston, P.M., J.M. Steckelberg, A.D. Hassen, M.S. Rose and M.E. Bolanda et al., 2000. Ciprofloxacin inhibition of experimental fracture healing. J. Bone Joint Surge. Am., 82: 161-173. PMID: 10682725
Hugo-Persson, M., 1996. Rupture of the Achilles tendon after ciproxine therapy. Lakartidningen, 93: 15201520. PMID: 8667750

Jagose, J.T., D.R. McGregor, G.R. Nind and R.R. Bailey, 1996. Achilles tendon rupture due to ciprofloxacin. N. Z. Med. J., 109: 471-472. PMID: 9006634

Kashida, Y. and M. Kato, 1997. Characterization of fluoroquinolone-induced Achilles tendon toxicity in rats: Comparison of toxicities of 10 fluoroquinolones and effects of anti-inflammatory compounds. Antimicrob. Agents Chemother., 14: 2389-2393. PMID: 9371338

Kato, M., 2008. Chondrotoxicity of quinolone antimicrobial agents. J. Toxicol. Pathol., 21: 123131. DOI: $10.1293 /$ tox. 21.123

Kelly, M., M. Dodds, J.S. Huntley and C.M. Robinson, 2004. Bilateral concurrent rupture of the Achilles tendon in the absence of risk factors. Hosp. Med., 65: 310-311. PMID: 15176151

Keutz, E.V., C. Ruhl, W.F. Drommer and M. Rosenbrunch, 2004. Effects of ciprofloxacin on joint cartilage in immature dogs immediately after dosing and after a 5-month treatment-free period. Arch. Toxicol., 78: 418-424.

Khaliq, Y. and G.G. Zhanel, 2003. Fluoroquinoloneassociated tendinopathy: A critical review of the literature. Clin. Infect. Dis., 36: 1404-1410. PMID: 12766835

Khazad, A. and A.J. Schwenk, 2005. Irrational Roots of Integers. College Math. J.

Kim, G.K., 2010. The risk of fluoroquinolone-induced tendinopathy and tendon rupture: What does the clinician need to know? J. Clin. Aesthetic. Dermatol., 3: 49-54. PMID: 20725547

Kotnis, R.A., J.C. Halstead and P.J. Hormbrey, 1999. Atraumatic bilateral Achilles tendon rupture: An association of systemic steroid treatment. J. Accid. Emerg. Med., 16: 378-379.

Lee, T.W. and J.F. Collins, 1992. Ciprofloxacin associated bilateral Achilles tendon rupture. Aust. N Z. J. Med., 22: 500-500.

Li, P., N.N. Cheng, B.V. Chen and Y.M. Weng, 2004. In vivo and in vitro chondrotoxicity of ciprofloxacin in juvenile rats. Arch. Pharmacol. Sin., 25: 1262-1266. PMID: 15456526

Li, P., N.N. Cheng, B.Y. Chen and Y.M. Wang, 1990. In vivo and in vitro chondrotoxicity of ciprofloxacin in juvenile rats. Acta Pharmacol. Sin., 25: 1262-1266. PMID: 15456526

Li, Q., S. Peng, Z. Sheng and Y. Wang, 2010. Ofloxacin induces oxidative damage to joint chondrocytes of Juvenile rabbits: Excessive production of reactive oxygen species. Lipid peroxidation and DNA damage. Eur. J. Pharmacol., 626: 146-153. PMID: 19818344 
Lica, K. and X. Zhao, 1997. DNA gyrase, topoisomerase IV and the 4-quinolones. Microbial. Mol. Rev., 61: 377-392. PMID: 9293187

Linden, P.K., R.C. Moellering, C.A. Wood, S.J. Rehm and J. Flaherty, 2001. Treatment of vancomycinresistant enterococcus faecium infections with quinupristin/dalfopristin. Clin. Infect. Dis., 33: 1816-1823.

Lode, H., K. Bomer and P. Koeppe, 1998. Pharmacodynamics of fluoroquinolones. Clin. Infect. Dis, 27: 33-39. PMID: 9675446

Lozo, J., M. Vukasinovic, I. Strahinic and A. Topisirovic, 2004. Characterization and antimicrobial activity of bacteriocin 217 produced by natural isolate Lactobacillus paracasei subsp. paracasei BGBUK216. J. Food Protect., 67: 2727-2734.

Maslanka, T., J.J. Jaroszewski and M. Chrostowska, 2004. Pathogenesis of quinolone-induced arthropathy: A review of hypotheses. Pol. J. Vet. Sci., 7: 323-331. PMID: 15633793

McEwan, S.R. and P.G. Davey, 1988. Ciprofloxacin and tenosynovitis. Lancet, 2: 900-900. PMID: 2902333

Mehra, A., R. Case and R.C. Croucher, 2004. Bilateral simultaneous spontaneous rupture of the Achilles tendon. Hosp. Med., 65: 308-309.

Mirovsky, Y., I. Pollack and A. Arlazoroff, 1995. Ciprofloxacin-associated bilateral acute achilles tendinitis. Harefuah, 129: 470-472. PMID: 8846955

Mitscher, L.A. and Z. Mao, 2003. Structure activity relationships of Quinolones. In: Fluoroquinolone Antibiotics, Ronald, A.R. and D.E. Low (Eds.)., Springer, Basel, Boston Berlin, ISBN-10: 3764365919, pp: 11-48.

Movin, T., A. Gad, P. Guntner, Z. Foldhazy and C. Rolf, 1997. Pathology of the Achilles tendon in association with ciprofloxacin treatment. Foot Ankle Int., 18: 297-299. PMID: 9167931

Olcay, E., O. Beytemur, F. Kaleagasioghi, T. Gulmez and Z. Mutlu, 2011. Oral toxicity of perfloxacin, norfloxacin, ofloxacin and ciprofloxacin: Comparison of biochemical and histopathological effects on Achilles tendon in rats. J. Toxicol. Sci., 36: 339-345.

Orava, S., M. Hume and J. Leppilahti, 1996. Bilateral Achilles tendon rupture: A report on two cases. Scand. J. Med. Sci. Spot., 6: 309-312. PMID: 8960654

Pantalone, A., M. Abafe, C. Diovidio, A. Carnevale and V. Salini, 2011. Diagnostic failure of ciprofloxacininduced spontaneous bilateral Achilles tendon rupture: Case-report and medical-legal considerations. Int. J. Immonopathol. Pharmacol., 24: 519-522. PMID: 21658328
Papich, M., 1998. Antibacterial drug therapy. Focus on new drugs. Vet. Clin. Pharm. Therapy, 28: 215-231. PMID: 9556846

Pauzaud, F., K. Bernard-Beaubois, M. Therenin, J.M. Warnet and G. Hoyem et al., 2004. In vitro discrimination of fluoroquinolones toxicity on tendon cells: Involvement of oxidative stress. J. Pharmacol. Exp. Ther., 308: 394-402. PMID: 14569066

Petersen, W. and H. Lapress, 1998. Insidious rupture of the Achilles tendon after ciprofloxacin-induced tendopathy. A case report. Unfallchirurgie, 101: 731-734. PMID: 9816984

Petri, W.A., 2001. Sulfonamides, TrimethroprimSulforne Thoxazole, Quinolones And Agents of Urinary Tract Infections. In: Goodman Gilman's the Pharmacological Buns of Therapeutics, Brunron L.L., (Ed.)., The MC Graw Hill Companies Inc., New York, pp: 1463-1476.

Pfister, K., D. Mazur, J. Vormann and R. Stahlman, 2007. Diminished ciprofloxacin-induced chrondrotoxicity by supplementation with magnesium and vitamin $\mathrm{E}$ in immature rats. Chemother, 51: 022-1027.

Pierfitte, C., R.J. Royer, N. Moore and B. Begaud, 2000. The link between sunshine and phototoxicity of sparfloxacin. Br. J. Clin. Pharmacol., 49: 609-612. DOI: $10.1046 / \mathrm{j} .1365-2125.2000 .00212 . \mathrm{x}$

Poon, C.C. and N.A. Sundaram, 1997. Spontaneous bilateral achilles tendon rupture associated with ciprofloxacin. Med. J. Aust., 166: 665-665.

Prasad, A.S., 1991. Discovery of human zinc deficiency and studies in an experimental human model. Am. J. Clin. Nutr., 53: 403-412.

Schuter, G., 1987. Ciprofloxacin review of potential toxicological effects. Am. J. Med., 82: 91-93.

Sendzik, J., M. Shakabaei, M. Schater-Korting, H. Lode and R. Stahlmann, 2010. Synergistic effects of dexamethasone and quinolones on human-derived tendon cells. Int. J. Antimicrob. Agents, 35: 366374. PMID: 20034766

Sendzik, J., M. Shakibaei, M. Schafer-Korting and R. Stahlmann, 2005. Fluoroquinolones cause changes in extracellular matrix, signalling proteins, metalloproteinases and caspase- 3 in cultured human tendon cells. Toxicology, 272: 24-36. PMID: 15890441

Shakibaei, M., K. Pfister, R. Schwabe, J. Vorman and R. Stahlmann, 2000. Ultrastructure of achilles tendons of rats treated with ofloxacin and fed a normal or magnesium-deficient diet. Antimicrob. Chemother., 44: 261-266. DOI: 10.1128/AAC.44.2.261-266.2000 
Shinohara, Y.T., S.A. Tasker, M.R. Wallace, K.E. Couch and P.E. Oison, 1997. What is the risk of Achilles tendon rupture with ciprofloxacin? J. Rheumat., 24: 238-239. PMID: 9002057

Short, P., N. Wilson and I. Erskin, 2006. Tendinitis: The Achilles heel of quinolones. Emerg. Med. J., 23: 6363. PMID: 17130584

Simonin, M.A., P. Gegout, M.A. Pottie, P. Gillet and P. Netter et al., 1999. Proteoglycan and collagen biochemical variations during fluoroquinoloneinduced chondrotoxicity in mice. Antimicrob. Agents. Chemother., 43: 2915-2921. PMID: 10582882

Stahlmann, R., 2000. Clinical toxicological aspects of fluoroquinolones. Toxicol. Lett., 127: 269-277. DOI: 10.1016/S0378-4274(01)00509-4

Stahlmann, R., 2003. Children as a special population at risk--quinolones as an example for xenobiotics exhibiting skeletal toxicity. Arch. Toxicol., 77: 7-11. PMID: 12491034

Stahlmann, R., C. Forster, M. Shakibaei, J. Vormann and T. Gunther et al., 1995. Magnesium deficiency induces joint cartilage lesions in juvenile rats which are identical to quinolone-induced arthropathy. Antimicrob. Agents Chemother., 39: 2013-2018. DOI: 10.1128/AAC.39.9.2013

Stahlmann, R., R. Schwabe, K. Pfister, E. Lozo and M. Shakkibaei et al., 1999. Supplementation with magnesium and tocopherol diminishes quinoloneinduced chondrotoxicity in immature rats. Drugs, 58: 393-394.

Stahlmann, R., S. Kuhner, M. Shakibaei, R. Schwabe and J. Flores et al., 2000. Chondrotoxicity of ciprofloxacin in immature Beagle dogs: Immunohistochemistry, electron microscopy and drug plasma concentrations. Arch. Toxicol., 73: 564-572.

Sub, B. and B. Lorber, 1995. Quinolones. Med. Clin. NA., 79: 869-894.
Thuong-Guyot, M., O. Domarle, J.J. Pocidalo and G. Hayem, 1994. Effects of fluoroquinolones on cultured articular chondrocytes flow cytometric analysis of free radical production. J. Pharmacol. Exp. Ther., 271: 1544-1549. PMID: 7996468

Tsai, A.G., D.F. Williamson and H.A. Glick, 2011. Direct medical cost of overweight and obesity in the USA: A quantitative systematic review. Obes Rev., 12: 50-61. DOI: 10.1111/j.1467-789X.2009.00708.X

Tsai, J., J.T. Lee, W. Wang, J. Zhang and H. Cho, 2008. Discovery of a selective inhibitor of oncogenic BRaf kinase with potent antimelanoma activity. PANS, 105: 3041-3046. DOI: 10.1073/pnas.0711741105

Water, I., M. Egerbacher, B. Wolfesberger and G. Seiberl, 1998. Confocal laser scanning microscopy of chondrocytes in vitro: Cytoskeletal changes after quinolone treatment. Scanning, 20: 511-575. PMID: 9857527

West, M.B. and P. Gow, 1998. Ciprofloxacin, bilateral Achilles tendonitis and unilateral tendon rupture--a case report. NZ. Med. J., 111: 18-19. PMID: 9484431

Williams, R.J., E. Altia, T.L. Wickiewicz and J.A. Hamafin, 2000. The effect of ciprofloxacin on tendon, paratenon and capsular fibroblast metabolism. Am. J. Sports Med., 28: 364-369. PMID: 10843129

Wilton, L.V., G.L. Pearce and R.D. Mann, 1996. A comparison of ciprofloxacin, norfloxacin, ofloxacin, azithromycin and cefixime examined by observational cohort studies. Br. J. Clin. Pharmacol., 41: 277-284. DOI: $\quad$ 10.1046/j.13652125.1996.03013.x 